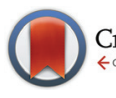

CrossMark \&lick for updates

Cite this: Dalton Trans., 2015, 44, 20441

Received 4th September 2015, Accepted 12th October 2015

DOI: $10.1039 / \mathrm{c} 5 \mathrm{dt} 03445 \mathrm{k}$

www.rsc.org/dalton

\section{High pressure synthesis of polar and non-polar cation-ordered polymorphs of $\mathrm{Mn}_{2} \mathrm{ScSbO}_{6} \dagger$}

\author{
E. Solana-Madruga, ${ }^{\star a}$ A. J. Dos santos-García, ${ }^{b}$ A. M. Arévalo-López, ${ }^{c}$ \\ D. Ávila-Brande, ${ }^{a}$ C. Ritter, ${ }^{d}$ J. P. Attfield ${ }^{c}$ and R. Sáez-Puche ${ }^{a}$
}

\section{Introduction}

Material properties are dependent upon atomic arrangement and the degree of order can be tailored by controlling the pressure-temperature conditions and bulk composition. At a given pressure $P$ and temperature $T$, there are different possible atomic arrangements that correspond to local minima of free energy, the lowest-energy conformation being the thermodynamically-stable phase. However, high pressure and high temperature (HPHT) synthesis conditions may favour higherenergy minima and metastable phases, making them kinetically stable under ambient conditions and therefore recoverable.

Amongst transition metal oxides, HPHT helps to stabilize unusual oxidation states and environments that result in useful properties, e.g. the room temperature ferromagnetic metal $\mathrm{CrO}_{2}{ }^{1}$ Moreover, HPHT promotes interesting structural mechanisms. The ilmenite (IL) $\mathrm{FeTiO}_{3}$, for example, crystallizes in the space group $R \overline{3}$ with Fe and Ti stacked into alternate layers along the $c$-axis. ${ }^{2}$ It transforms into an unquenchable distorted perovskite (Pv) structure (space group $\mathrm{Pbnm})$ at $16 \mathrm{GPa}$ and converts back into a polar $\mathrm{LiNbO}_{3}$-type

\footnotetext{
${ }^{a}$ Dpto. Quimica Inorgánica, Universidad Complutense de Madrid, Av. Complutense sn, 28040-Madrid, Spain.E-mail: esolana@ucm.es

${ }^{b}$ Dpto. Ingeniería Mecánica, Quimica y Diseño Industrial, Universidad Politécnica de Madrid, C/ Ronda de Valencia 3, 28012-Madrid, Spain

${ }^{c}$ Centre for Science at Extreme Conditions and School of Chemistry, University of Edinburgh, Mayfield Road, EH9 3JZ, UK

${ }^{d}$ Institut Laue-Langevin, 38042 Grenoble Cedex, France

$\dagger$ Electronic supplementary information (ESI) available. CCDC 1428909-1428911. For ESI and crystallographic data in CIF or other electronic format see DOI: $10.1039 / \mathrm{c} 5 \mathrm{dt} 03445 \mathrm{k}$
}

(LN, space group $R 3 c$ ) with Fe and Ti cations being ordered in the same layers. ${ }^{3,4}$

Moreover, $\mathrm{ABO}_{3}$ oxides with $\mathrm{Mn}^{2+}$ on the A site are of fundamental interest due to the electronic and magnetic phenomena that emerge from the coupling of spin, charge and orbital degrees of freedom. HPHT conditions are often needed to stabilize $\mathrm{MnBO}_{3}$ materials, e.g. perovskite-type $\mathrm{MnVO}_{3}$ with an incommensurate magnetic structure and metallic conductivity, ${ }^{5}$ and $\mathrm{LiNbO}_{3}$-type $\mathrm{MnTiO}_{3}$-II with a weak ferromagnetism through anisotropic exchange interactions. ${ }^{6}$ The use of HPHT on ordered quaternary systems $\left(\mathrm{AA}^{\prime} \mathrm{B}_{2} \mathrm{O}_{6}\right.$ or $\left.\mathrm{A}_{2} \mathrm{BB}^{\prime} \mathrm{O}_{6}\right)$ is a relatively less explored area with only a few reports. ${ }^{7,8}$ Amongst these, $\mathrm{Mn}_{2} \mathrm{FeB}^{\prime} \mathrm{O}_{6}\left(\mathrm{~B}^{\prime}=\mathrm{Ta}\right.$ and $\left.\mathrm{Nb}\right)$ with a LN-type structure ${ }^{9}$ or $\left(\mathrm{B}^{\prime}=\mathrm{Mo}, \mathrm{W}\right)$ with the $\mathrm{Ni}_{3} \mathrm{TeO}_{6}$-type $(\mathrm{NTO})^{10,11}$ order shows polar and magnetic properties, while $\mathrm{Mn}_{2} \mathrm{BSbO}_{6}(\mathrm{~B}=\mathrm{Fe}$ and $\mathrm{Cr})$ shows polymorphism between the IL- and double perovskite-types (DPv) depending on whether they are synthesized at moderate (3-5 GPa) or higher pressures (5.5-8 GPa). ${ }^{12,13}$

In this work, we present the order-disorder effects on the high pressure polymorphs of $\mathrm{Mn}_{2} \mathrm{ScSbO}_{6}$ oxide. Mn and Sc cations are randomly distributed in a corundum-related type structure when this material is synthesized under ambient conditions and it shows no long-range magnetic ordering. ${ }^{14}$ The double perovskite (DPv) can be achieved with pressures higher than $10 \mathrm{GPa}$, but below $5.5 \mathrm{GPa}$ the polar NTO-structure is obtained. NTO_ $\mathrm{Mn}_{2} \mathrm{ScSbO}_{6}$ shows an unusual ferrimagnetism due to partial substitution of non-magnetic $\mathrm{Sc}^{3+}$ at just one of the two $\mathrm{Mn}^{2+}$ sites, whereas DPv_ $\mathrm{Mn}_{2} \mathrm{ScSbO}_{6}$ is antiferromagnetic. Combined X-ray and powder neutron diffraction refinements and electron micro-diffraction experiments confirm the non-centrosymmetry of the NTO_ $\mathrm{Mn}_{2} \mathrm{ScSbO}_{6}$ polymorph allowing a structural polarization that is predicted to 
be $28.3 \mu \mathrm{C} \mathrm{cm}^{-2}$ at room temperature. These results demonstrate that cation ordering can be induced by high pressure synthesis providing access to new metastable phases with unusual properties including multiferroicity. We also show that site selective disorder provides an unusual way to induce ferrimagnetism.

\section{Experimental section}

$\mathrm{Mn}_{2} \mathrm{ScSbO}_{6}$ was previously synthesized at ambient pressure by Kosse et al. ${ }^{15,16}$ and subsequently studied by Ivanov et al. ${ }^{14}$ NPD studies showed a random distribution of Mn and Sc crystallizing in a corundum-related structure $(R \overline{3})$ with no longrange magnetic ordering. We prepared a sample at $1373 \mathrm{~K}$ and Supplementary Fig. 1 (SF1 $\dagger$ ) shows the Rietveld fit to the XRD pattern that agrees with a random cation distribution.

Both double perovskite and $\mathrm{Ni}_{3} \mathrm{TeO}_{6}$-type polymorphs were synthesized under high pressure and high temperature conditions. The precursor, prepared by grinding stoichiometric amounts of $\mathrm{Mn}_{2} \mathrm{O}_{3}, \mathrm{Sc}_{2} \mathrm{O}_{3}$ and $\mathrm{Sb}_{2} \mathrm{O}_{3}$ oxides, was treated at 12 GPa and $1473 \mathrm{~K}$ for 20 minutes in a Walker-type multianvil apparatus for the preparation of the perovskite phase. The application of $5.5 \mathrm{GPa}$ and $1523 \mathrm{~K}$ for 35 minutes in a belt-type press led to the formation of the $\mathrm{Ni}_{3} \mathrm{TeO}_{6}$ polymorph. In both cases, the sample was quenched by rapid cooling and the pressure was progressively released down to ambient conditions.

The crystal structures were first characterized by Rietveld refinement of powder X-ray diffraction collected on a Phillips X'Pert Pro Alfa 1 diffractometer using $\mathrm{Cu} \quad \mathrm{K} \alpha_{1}$ radiation, equipped with a Ge (111) monochromator working in BraggBrentano geometry. The diffractograms were collected between 10 and 120 degrees with a step size of $0.017^{\circ}$.

For transmission electron microscopy (TEM) studies, samples were ground in $n$-butyl alcohol and ultrasonically dispersed. A few drops of the resulting suspension were deposited on a carbon-coated grid. The study of reciprocal space by selected area electron diffraction (SAED) and microdiffraction was carried out using a JEOL JEM2100 microscope operating at $200 \mathrm{kV}$ with a double tilt $\left( \pm 42^{\circ}\right)$ goniometer. High resolution transmission electron microscopy (HRTEM) and electron energy loss spectroscopy (EELS) experiments were performed with a JEOL JEM $3000 \mathrm{~F}$ microscope operating at $300 \mathrm{kV}$ (double tilt $\left( \pm 25^{\circ}\right)$ point resolution $0.17 \mathrm{~nm}$ ), fitted with an energy-dispersive X-ray spectroscopy (XEDS) microanalysis system (OXFORD INCA) and an ENFINA spectrometer with an energy resolution of $1.3 \mathrm{eV}$.

Simulations of the HRTEM images were performed with the software MacTempas $\mathrm{X},{ }^{17}$ using the refined structures from neutron diffraction data. The oxidation state of $\mathrm{Mn}$ in both materials was determined by EELS with the relationship between the white-line intensity ratio $\left(L_{3} / L_{2}\right)$ and the oxidation state. ${ }^{18}$

Neutron powder diffraction (NPD) patterns were collected at $300 \mathrm{~K}$ on a high resolution D2B diffractometer (Institut LaueLangevin, Grenoble) between 0 and $160^{\circ}$ with a step-width of $0.05^{\circ}$, using a neutron wavelength $\lambda=1.594 \AA$ and a standard He cryostat. The NTO polymorph was also cooled down to $4 \mathrm{~K}$, where a long scan was measured under the same conditions. The evolution of the magnetic structures was studied using the sequential patterns collected at each degree from $3 \mathrm{~K}$ up to $55 \mathrm{~K}$ on the high intensity D1B instrument. Each pattern was measured in the angular range $0^{\circ} \leq 2 \theta \leq 130^{\circ}$ with a step-width of $0.1^{\circ}$ using $\lambda=2.520 \AA$. Long scans were also taken at $3 \mathrm{~K}$.

The nuclear structures were refined from room temperature D2B data, using Rietveld analysis through the FullProf software package $^{19}$ and considering a Thomson-Cox-Hastings function to optimize the shape of the peaks. The magnetic symmetry analysis of the low temperature D1B data was performed by means of the program BasIreps. ${ }^{20}$

Magnetic susceptibility measurements were performed on a Quantum Design XL-MPMS SQUID magnetometer under DC Zero-Field-Cooling (ZFC) and Field-Cooling (FC) conditions in the temperature range $2 \mathrm{~K}<T<300 \mathrm{~K}$ under a magnetic field of 3 kOe. Magnetization dependence on the magnetic field strength was studied at 5, 20, 40 and $90 \mathrm{~K}$ for the perovskite polymorph up to $5 \mathrm{~T}$ and at 2, 20 and $40 \mathrm{~K}$ for the NTO-type oxide up to $7 \mathrm{~T}$.

\section{Results and discussion}

\section{(A) NTO_Mn $\mathrm{ScSbO}_{6}$}

Structural characterization. The lower pressure polymorph of $\mathrm{Mn}_{2} \mathrm{ScSbO}_{6}$ can be obtained at $5.5 \mathrm{GPa}$ and $1523 \mathrm{~K}$. Fig. 1 (bottom) shows the XRD diffraction pattern of this material. It can be indexed in a hexagonal cell with $a=5.34186$ (5) $\AA$ and $c=14.0603$ (2) A cell parameters. This cell is indicative of a corundum-type related structure, as observed in other $\mathrm{Mn}_{2} \mathrm{BB}^{\prime} \mathrm{O}_{6}$ oxides. $^{9-13}$ The presence of the (003) and (101) reflections (inset Fig. 1-bottom) demonstrates the absence of a $c$-glide plane and therefore excludes the corundum- $(R \overline{3} c)$ and LN-type $(R 3 c)$ structures, suggesting an ilmenite-type ordering $(R \overline{3})$. Rietveld refinement was performed with the $\mathrm{IL}_{-} \mathrm{Mn}_{2} \mathrm{FeSbO}_{6}$ structure as a starting model. The refinement converged to $R_{\mathrm{wp}}$ $=11.3 \%$ and $R_{\mathrm{p}}=6.24 \%$ agreement factors.

However, NPD experiments on the same compound revealed no intensity for the (003) and (101) reflections (Fig. 1top) and the IL-type model obtained from XRD refinement showed a poor fit to the neutron data. The data can be fitted assuming a LN-type ordering $(R 3 c)$ with $R_{\mathrm{wp}}=4.40 \%$ and $R_{\mathrm{p}}=$ $3.41 \%$ agreement factors.

In order to clarify this situation, electron microscopy experiments were performed. The SAED patterns exhibit the reflection conditions: $h k i l:-h+k+l=3 n$, hki0: $-h+k=3 n$, 000l: $l=$ $3 n, h-h 00: h=3 n, h-h 0 l: h+l=3 n$ and the only feasible space groups are $R \overline{3}$ and $R 3$ (Fig. $2 \mathrm{a}-\mathrm{c}$ ).

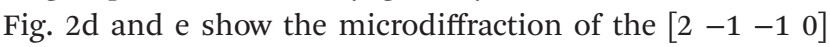
zone axis. The no periodicity difference between the zero and first order Laue zones (ZOLZ and FOLZ) indicates the absence of a $c$-glide plane, in agreement with XRD. Moreover, the analysis of the whole pattern (WP) of the $\left[\begin{array}{llll}2 & -1 & -1 & 0\end{array}\right]$ and $\left[\begin{array}{llll}0 & 1 & -1 & 0\end{array}\right]$ 

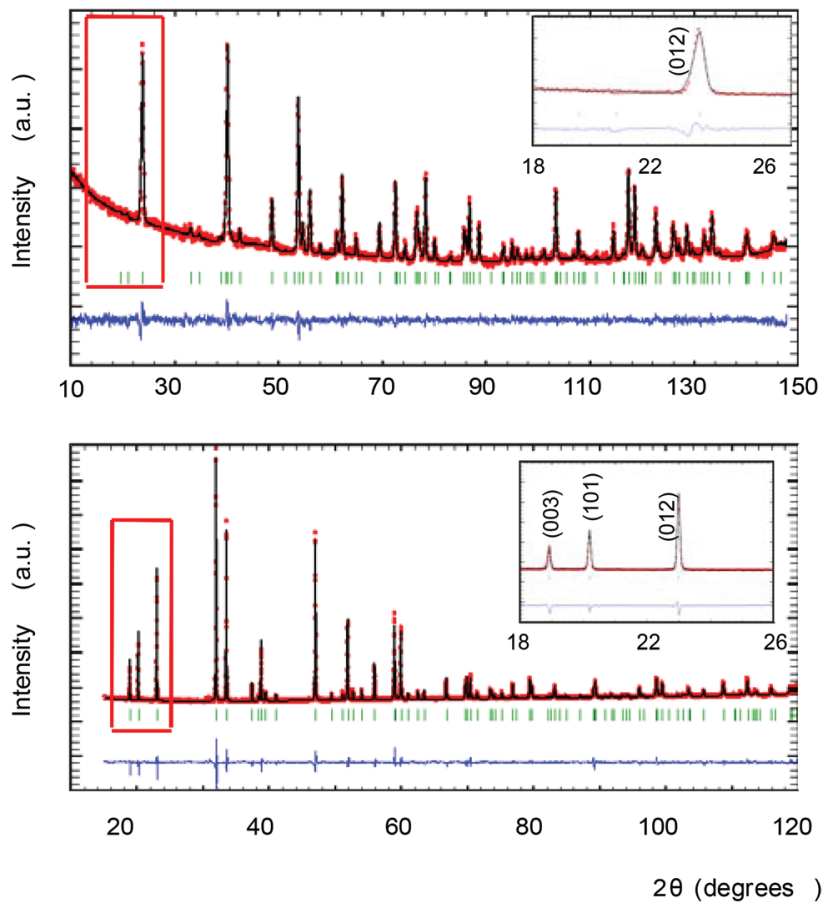

Fig. 1 Rietveld refinements of the nuclear structure of NTO_ $\mathrm{Mn}_{2} \mathrm{ScSbO}_{6}$ from room temperature NPD profile collected using a D2B diffractometer (top) and XRD data (bottom). The insets show an enlargement of the low angle range identified with the red rectangles, where reflections (003) and (101) are observed in the XRD pattern although they are not seen in the NPD profile, as discussed in the text.

zone axes (Fig. 2f) unequivocally distinguishes between $R \overline{3}$ (two-fold axis) and $R 3$ (no symmetry). ${ }^{21}$

A model with $R 3$ symmetry was then tested. In order to break the inversion centre of the IL-type model, we assumed a complete ordering within the (00z)-layers between Mn1/Sc and $\mathrm{Mn} 2 / \mathrm{Sb}$; this cation arrangement is adopted in the $\mathrm{Ni}_{3} \mathrm{TeO}_{6}$ type structure with the polar $R 3$ space group. The NTO-type structure has also been observed in the related compounds $\mathrm{Mn}_{2} \mathrm{FeMoO}_{6}$ and $\mathrm{Ni}_{2} \mathrm{ScSbO}_{6}{ }^{10,22}$ The refinement converged smoothly; Fig. 1 shows the Rietveld refinements of the NPD (top) and XRD (bottom) data for the NTO_ $\mathrm{Mn}_{2} \mathrm{ScSbO}_{6}$ model and Table 1 summarizes the structural details and agreement factors resulting from this combined refinement.

The final model clarifies the apparent contradiction between the XRD and NPD results. The cation arrangement of the $R 3$ NTO-type structure is illustrated in Fig. 3 (left). The (00z) layers alternate between $\mathrm{Mn} 1 / \mathrm{Sc}$ and $\mathrm{Mn} 2 / \mathrm{Sb}$ composition. For XRD, Sc $(Z=21)$ and $\mathrm{Mn}(Z=25)$ look similar when compared with the consecutive layer of $\mathrm{Mn}$ and $\mathrm{Sb}(Z=50)$. Therefore, IL-type ordering is apparent and the (003) and (101) reflections are observable. On the other hand, the difference in neutron scattering lengths for $\mathrm{Mn}, \mathrm{Sc}$ and $\mathrm{Sb}(-3.73,12.29$ and $5.57 \mathrm{fm}$ respectively) breaks the apparent inversion centre, as the (012) planes are alternatively constituted by $\mathrm{Mn}$ or by $\mathrm{Sc} / \mathrm{Sb}$, which makes the scattering distribution resemble that of an LN-type. For clarity, both IL- and LN-type orders are high-
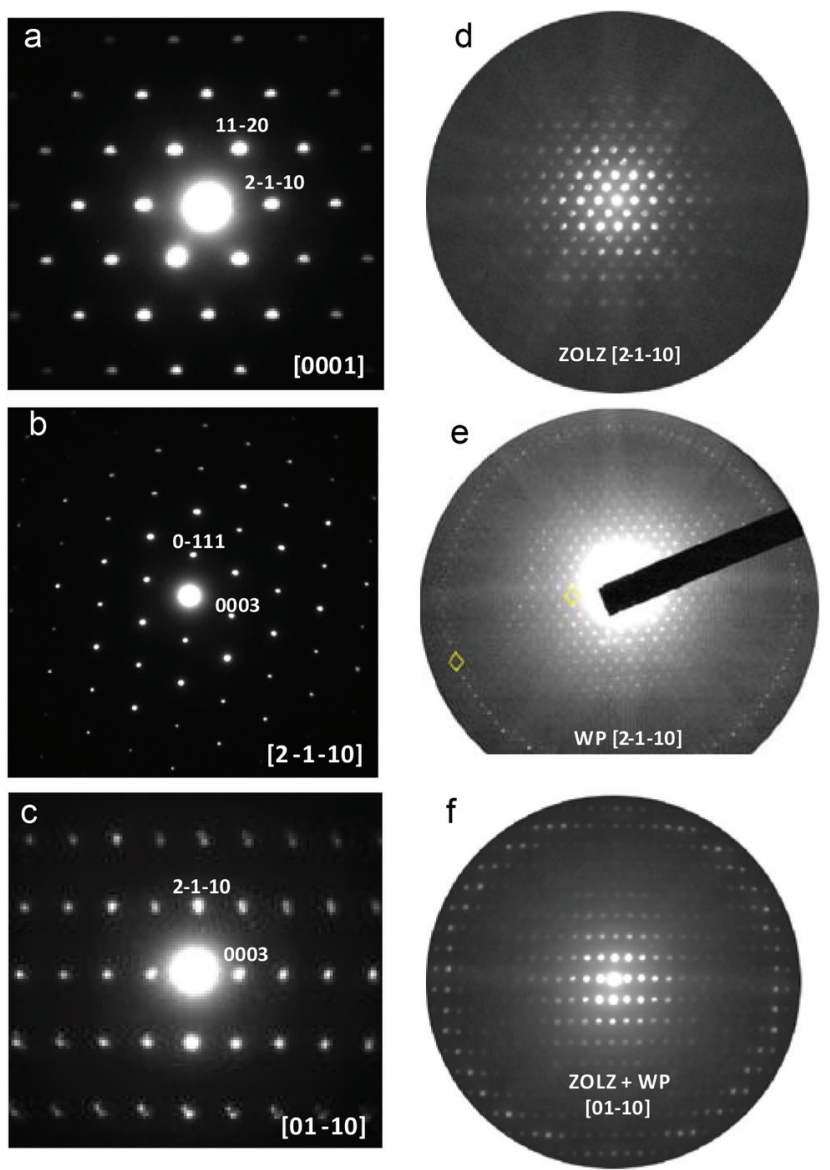

Fig. 2 SAED patterns for NTO_Mn ${ }_{2} \mathrm{ScSbO}_{6}$ along the [l $\left.\begin{array}{llll}0 & 0 & 0 & 1\end{array}\right],\left[\begin{array}{llll}2 & -1 & -1 & 0\end{array}\right]$ and [0 $1-10$ ] zone axes (a-c, respectively) and microdiffraction patterns $(d-f)$ along the [2 - $1-10]$ and [0 $1-1$ - 0 ] zone axes.

lighted in Fig. 3 (left) with black and red dashed lines respectively. In conclusion, the combination of SAED, NPD and XRD techniques unequivocally leads to the true NTO-type structure of this compound.

EELS analysis performed on several crystallites yields a +2 oxidation state for Mn (see ESI Fig. SF2a $\dagger$ ). A structural HRTEM image along the $\left[\begin{array}{ccc}2-1 & -1 & 0\end{array}\right]$ zone axis (SF2 $b_{\dagger} \dagger$ ) shows a well ordered material and the absence of extended defects.

The structure of NTO_ $\mathrm{Mn}_{2} \mathrm{ScSbO}_{6}$ can be described as a corundum derivative with $(00 z)$ layers alternatively occupied by $\left[\mathrm{Mn} \mathrm{O}_{6}\right] /\left[\mathrm{ScO}_{6}\right]$ and $\left[\mathrm{Mn} 2 \mathrm{O}_{6}\right] /\left[\mathrm{SbO}_{6}\right]$ edge sharing octahedra. The order is such that the polyhedra sharing faces along the $c$ axis are always Mn1 against $\mathrm{Sb}$ and Sc against Mn2 (see Fig. 3 left). All site occupancies were tested by allowing cation exchange among the different sites, but the only significant result was found between Sc and Mn2 positions and the final refinement converged to a value of 12.3 (2)\% of anti-site mixing. The insertion of this small amount of $\mathrm{Sc}^{3+}$ in the Mn2 site decreases its octahedral distortion from $5.5 \times 10^{-3}$ observed for Mn1 down to $1.7 \times 10^{-3}$, while the observed $\Delta\left[\mathrm{ScO}_{6}\right]$ value remains $1.1 \times 10^{-4}$ as that observed for $\left[\mathrm{SbO}_{6}\right]$. 
Table 1 Crystallographic parameters, selected interatomic distances ( $(\AA)$ and angles $\left(^{\circ}\right)$ for NTO_ $\mathrm{Mn}_{2} \mathrm{ScSbO}_{6}$ from the combined refinement of room temperature NPD and XRD data in the $R 3$ space group ${ }^{a}$

$a=5.3419(5)(\AA), \quad c=14.0603(2)(\AA)$

\begin{tabular}{|c|c|c|c|c|c|}
\hline Atom & Site & $x$ & $y$ & $z$ & Occ \\
\hline $\mathrm{Mn} 2 / \mathrm{Sc}$ & $3 \mathrm{a}$ & $2 / 3$ & $1 / 3$ & $0.3423(8)$ & $0.877(2) / 0.123(2)$ \\
\hline Sc/Mn2 & $3 a$ & 0 & 0 & $0.2224(8)$ & $0.877(2) / 0.123(2)$ \\
\hline $\mathrm{O} 1$ & $9 b$ & $0.641(1)$ & $0.695(2)$ & $0.2926(7)$ & 1 \\
\hline $\mathrm{O} 2$ & $9 b$ & $0.998(2)$ & $0.622(2)$ & $0.4555(5)$ & 1 \\
\hline$\left(\mathrm{Mn} 1-\mathrm{O}_{1}\right) \times 3$ & $2.42(1)$ & $\left(\mathrm{Mn} 2-\mathrm{O}_{1}\right) \times 3$ & $2.12(1)$ & $\Delta\left[\mathrm{Mn}_{1} \mathrm{O}_{6}\right]$ & $5.5 \times 10^{-3}$ \\
\hline$\left(\mathrm{Sb}-\mathrm{O}_{2}\right) \times 3$ & $1.98(1)$ & $\left(\mathrm{Sc}-\mathrm{O}_{2}\right) \times 3$ & $2.18(1)$ & $\Delta\left[\mathrm{ScO}_{6}\right]$ & $1.1 \times 10^{-4}$ \\
\hline$<$ Mn1-O1-Mn2> & \multicolumn{2}{|c|}{$121.0(4)$} & \multicolumn{2}{|c|}{$<\mathrm{Mn} 1-\mathrm{O} 2-\mathrm{Mn} 2>$} & $118.4(3)$ \\
\hline $\operatorname{BVS}(\operatorname{Mn} 1)^{b}=1.89$ & \multicolumn{2}{|c|}{$\mathrm{BVS}(\mathrm{Sb})=5.18$} & \multicolumn{2}{|c|}{ BVS $(\mathrm{Mn} 2)=2.01$} & $\operatorname{BVS}(\mathrm{Sc})=2.93$ \\
\hline
\end{tabular}

${ }^{a}$ Fitting residuals: $R_{\mathrm{p}}=3.04 \%, R_{\mathrm{wp}}=3.86 \%, R_{\mathrm{B}}=6.18 \%$ and $R_{\mathrm{F}}=5.04 \%,{ }^{b} V_{i}=\sum_{j} S_{i j} i=\exp \left(r_{0}-r_{i j} / 0.37\right)$. Values calculated using $r_{i j}=1.79 \AA$ for $\mathrm{Mn}^{2+}, 1.849 \AA$ for $\mathrm{Sc}^{3+}$ and $1.942 \AA$ for $\mathrm{Sb}^{5+}$. Polyhedral distortions calculated from $\Delta=1 / n \times \sum\left[\left(d_{\mathrm{i}}-d_{\mathrm{av}}\right) / d_{\mathrm{av}}\right]^{2}$.

The displacement of the different cations from their ideal sites with reference to the oxygen octahedra are labeled in Fig. 3(left). Mn cations show larger displacements in comparison with the $\mathrm{d}^{0} \mathrm{Sc}$ or $\mathrm{d}^{10} \mathrm{Sb}$, and in accordance with the greater distortion found for their polyhedra (see Table 1).

The theoretical value of polarization along the $z$ axis has been calculated from $P=\sum_{j} q_{j} u_{j}^{z}$, where $q_{j}$ stands for the charge over a generic $j$ ion and $u_{j}^{z}$ for its displacement along the $z$ axis, resulting in $P_{\text {expected }}=28.3 \mu \mathrm{C} \mathrm{cm}^{-2}$. This estimate neglects the effects of $\mathrm{Mn} / \mathrm{Sc}$ disorder. Attempts were made to measure the experimental polarization, using a modified Sawyer-Tower circuit. $^{23}$ Due to the low resistivity, sample polarization is masked by conducting effects and typical inconclusive P vs. E ellipse-shaped cycles were obtained.

Magnetic behaviour and magnetic structure. The magnetic susceptibility of NTO_Mn $\mathrm{ScSbO}_{6}$, represented in Fig. 4a with its inverse, follows a Curie-Weiss behaviour above $75 \mathrm{~K}$ with a
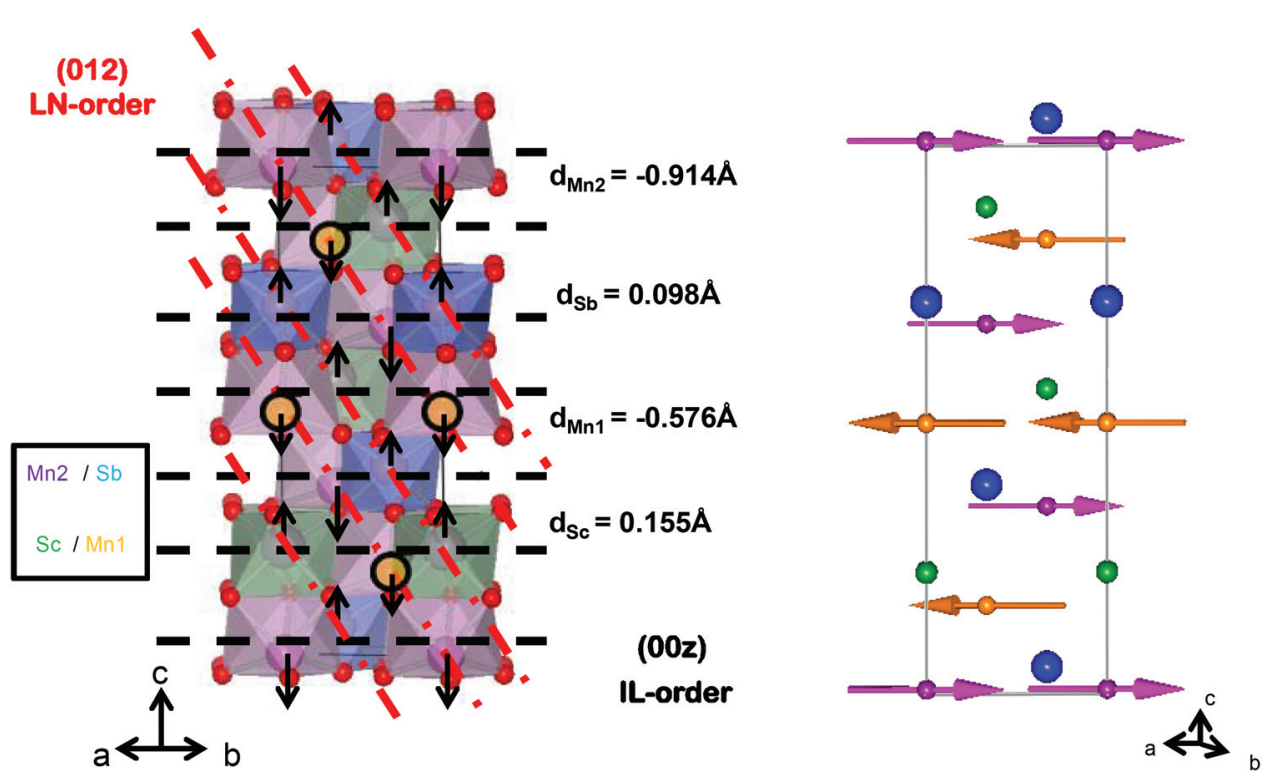

Fig. 3 Unit cell of the nuclear (left) and magnetic (right) structures of the NTO polymorph of $\mathrm{Mn}_{2} \mathrm{ScSbO}_{6}$. In both, Sc is depicted in green, Sb in blue and the two different sites for $\mathrm{Mn}$ cations are identified with purple and orange spheres. $\left[\mathrm{MO}_{6}\right]$ octahedral coordination can be observed in the left, where both $\mathrm{Mn}$ octahedra are purple to facilitate the visualization of LN-type order. (00z) and (012) layers showing the apparent IL and LN-type orders, respectively, are labeled as black and red dashed lines. The black arrows show the cationic displacements $\left(d_{M}\right)$ along the $c$ axis. Purple and orange arrows in the right part of the figure indicate the modulus and direction of $\mathrm{Mn}^{2+}$ spins located in $\mathrm{Mn} 1$ and $\mathrm{Mn} 2$ sites respectively. 
calculated effective moment of $\mu_{\mathrm{NTO}}=6.1 \mu_{\mathrm{B}}$ per $\mathrm{Mn}^{2+}$ and $\theta_{\text {NTO }}=-138 \mathrm{~K}$. A ferro- or ferri-magnetic transition is observed at $T_{\mathrm{C}}=42 \mathrm{~K}$. There is no significant divergence between ZFC and FC branches, and field dependent magnetization measurements (inset in Fig. 4a and SF3† top) show that NTO_ $\mathrm{Mn}_{2} \mathrm{ScSbO}_{6}$ is a very soft ferrimagnet with a saturated magnetization of $0.6 \mu_{\mathrm{B}}$ per f.u. at $2 \mathrm{~K}$.

From the structural point of view, $\mathrm{Mn}^{2+}$ cations are located in different octahedral sites within (00z) layers and are connected to each other through sharing corners. This allows cooperative AFM Mn-O-Mn superexchange interactions that are governed by $<\mathrm{Mn} 1-\mathrm{O} 1-\mathrm{Mn} 2>=121.0(4)^{\circ}$ and $<\mathrm{Mn} 1-\mathrm{O} 2-$ Mn2 $>=118.4(3)^{\circ}$ angles.

The $4 \mathrm{~K}$ NPD data collected for NTO_Mn ${ }_{2} \mathrm{ScSbO}_{6}$ at D2B instrument are shown in Fig. 4b. The magnetic structure of this polymorph (Fig. 3 right) was determined from the fit to these data, where $27^{\circ}-30^{\circ}$ and $46^{\circ}-48^{\circ}$ angular ranges are excluded due to the presence of peaks arising from the sample holder. Additional magnetic neutron diffraction peaks are
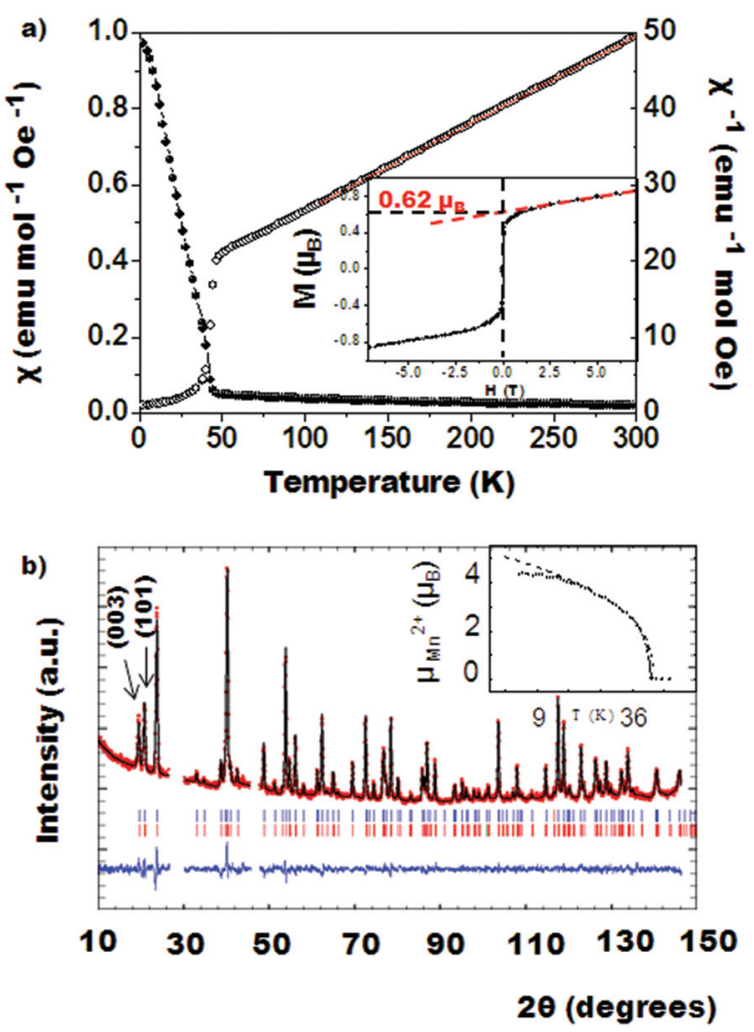

Fig. 4 (a) NTO_Mn ${ }_{2} \mathrm{ScSbO}_{6}$ magnetic susceptibility as a function of temperature under an applied magnetic field of $3 \mathrm{kOe}$ (black circles). Reciprocal susceptibility (open circles) is indexed on the right axis and fitted (red line) to the Curie-Weiss law. Inset in (a) shows the magnetization loop measured at $2 \mathrm{~K}$ showing the saturated magnetization per formula unit. (b) Rietveld refinement of the magnetic structure from the 4 K NPD pattern. The main magnetic peaks (003) and (101) are indexed. Inset shows the temperature dependence of the magnetic moment of $\mathrm{Mn}^{2+}$ with a fit (dashed line) to a critical law $\mu(T)=\mu(0) \times\left[1-\left(T / T_{C}\right)\right]^{\beta}$, as described in the text. observed below $T_{\mathrm{C}}=42 \mathrm{~K}$, the most intense being (003) and (101), which can be indexed with the propagation vector $k=$ $\left[\begin{array}{lll}0 & 0 & 0\end{array}\right]$. Fits to the magnetic peaks confirm a FM coupling of Mn1 spins within $00 z$ layers, where spins are confined to the $a b$ plane, but antiferromagnetically coupled with the Mn2 cations of the adjacent layers. The refined moment per $\mathrm{Mn}^{2+}$ cation is 4.62 (3) $\mu_{\mathrm{B}}$. The spin order and Mn site occupancies observed by NPD reveal the origin of the ferromagnetic behavior of $\mathrm{NTO}_{-} \mathrm{Mn}_{2} \mathrm{ScSbO}_{6}$. Antiparallel Mn1 and Mn2 spins are symmetry-inequivalent and so a small uncompensated ferromagnetic moment is expected, but this is greatly enhanced by site-selective disorder as Mn1 sites are fully occupied by $\mathrm{Mn}^{2+}$ spins, but $12.3 \%$ of Mn2 sites are substituted by non-magnetic $\mathrm{Sc}^{3+}$. The predicted uncompensated moment $\left(12.3 \% \times 4.62 \mu_{\mathrm{B}}\right.$ $=0.57 \mu_{\mathrm{B}}$ ) is in excellent agreement with the observed magnetization of $0.6 \mu_{\mathrm{B}}$. Hence, the ferromagnetic component arises from an unusual site-selective substitution of non-magnetic $\mathrm{Sc}^{3+}$ at just one of the two $\mathrm{Mn}^{2+}$ sites. A similar ferrimagnetism due to selective disorder has been recently observed in $\mathrm{DPv}$ type $\mathrm{La}_{3} \mathrm{Ni}_{2} \mathrm{SbO}_{9} \cdot{ }^{24}$ Magnetic diffraction from the ferromagnetic component in NTO_ $\mathrm{Mn}_{2} \mathrm{ScSbO}_{6}$ is expected to be very weak and is not observed in the difference pattern between NPD data sets collected at $50 \mathrm{~K}$ and $3 \mathrm{~K}$ on the D1B instrument (SF4† lower panel).

The evolution of the magnetic moment below the Curie temperature (inset on Fig. 4b), was fitted to a critical law $\mu(T)=$ $\mu(0) \times\left[1-\left(T / T_{\mathrm{C}}\right)\right]^{\beta}$ in the $\left(T_{\mathrm{C}} / 2\right)<T<T_{\mathrm{C}}$ temperature range between $21 \mathrm{~K}$ and $41 \mathrm{~K}$. It led to the parameters $T_{\mathrm{C}}=42.0 \mathrm{~K}$ and $\beta=0.37$, consistent with $3 \mathrm{D}$ Heisenberg behaviour for which $\beta=0.36$ is predicted.

Overall, NTO_ $\mathrm{Mn}_{2} \mathrm{ScSbO}_{6}$ is notable as a potential multiferroic material, with ferroelectricity arising from the acentric cation order in the NTO-type arrangement, and ferrimagnetism from the uncompensated antiparallel order of $\mathrm{Mn}^{2+}$ spins enhanced by site-selective $\mathrm{Mn} / \mathrm{Sc}$ disorder. This material seems to be a type I multiferroic as the two orders are decoupled. The magnetoelectric coupling between the electrical and magnetic polarizations may be small as they are orthogonal, being respectively parallel and perpendicular to the $c$-axis, although this could lead to an unusual switching mechanism as predicted for $\mathrm{LiNbO}_{3}$-type $\mathrm{MnTiO}_{3}$-II. ${ }^{6}$

\section{(B) $\mathrm{DPv} \_\mathrm{Mn}_{2} \mathrm{ScSbO}_{6}$}

Structural characterization. As in other related $\mathrm{Mn}_{2} \mathrm{BSbO}_{6}$ compounds ( $\mathrm{B}=\mathrm{Fe}, \mathrm{V}, \mathrm{Cr}, \mathrm{Ga}, \mathrm{Al}),{ }^{25}$ high pressure synthesis was needed to stabilize the small $\mathrm{Mn}^{2+}$ cation in the highly coordinated A site of the perovskite structure.

Fig. 5a (top) shows the Rietveld refinement of NPD data of $\mathrm{DPv} \_\mathrm{Mn}_{2} \mathrm{ScSbO}_{6}$ collected at room temperature. It was fitted using the $\mathrm{Mn}_{2} \mathrm{BSbO}_{6}(\mathrm{~B}=\mathrm{Fe}, \mathrm{Cr})^{12,13}$ structure as the starting model and crystallizes in the $P 2_{1} / n$ monoclinic space group

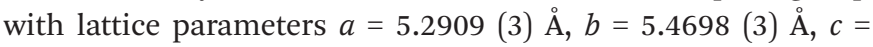
7.7349 (5) $\AA$ and $\beta=90.165$ (6) ${ }^{\circ}$. The resulting crystallographic parameters, interatomic distances and bond angles are summarized on Table 2. Anti-site disorder was found between Sc and $\mathrm{Mn}$, resulting in $9.1 \%$ of the A-site occupied by $\mathrm{Sc}^{3+}$. The 

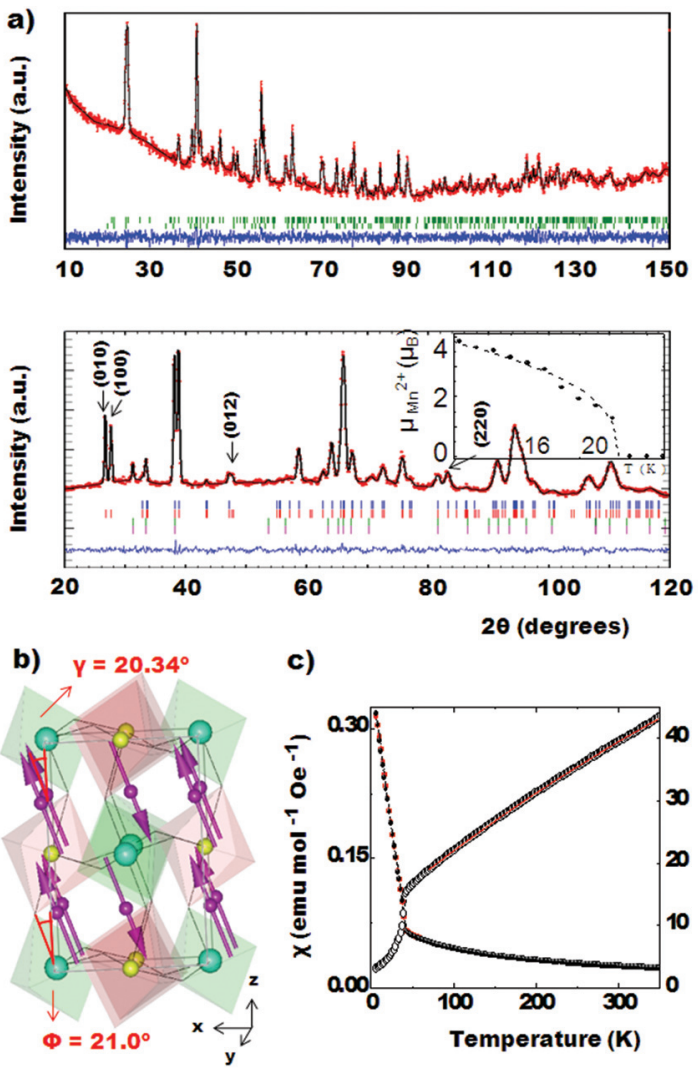

c)

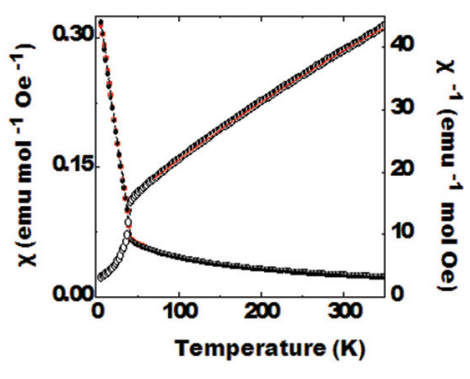

Fig. 5 (a) Rietveld refinements of room temperature (top) and $3 \mathrm{~K}$ (bottom) NPD patterns. $20.1 \%$ of the NTO polymorph is included as the secondary phase. First and second rows of Bragg positions in the lower patterns are the nuclear and (indexed) magnetic phases of the perovskite polymorph and the third and fourth rows are the nuclear and magnetic structures of the NTO_ $\mathrm{Mn}_{2} \mathrm{ScSbO}_{6}$ secondary phase. The inset in the lower panel shows the temperature dependence of the magnetic moment of $\mathrm{Mn}^{2+}$ fitted (dashed line) to the critical law described in the text. (b) Nuclear and magnetic structures of $\mathrm{DPv}_{-} \mathrm{Mn}_{2} \mathrm{ScSbO}_{6} .\left[\mathrm{ScO}_{6}\right]$ octahedra are depicted in red and $\left[\mathrm{SbO}_{6}\right]$ in green; $\mathrm{Mn}^{2+}$ cations, are shown as purple spheres with arrows indicating their magnetic moments. (c) Direct and reciprocal susceptibility (dark and empty circles respectively) as a function of temperature. secondary phase included in the refinement of $\mathrm{DPv}_{-} \mathrm{Mn}_{2} \mathrm{ScSbO}_{6}$ is the NTO polymorph formed at lower pressures during the synthesis reaction. A $20.1 \%$ of this phase has been found to crystallize within this high pressure compound. The presence of such a high proportion of $\mathrm{NTO}_{-} \mathrm{Mn}_{2} \mathrm{ScSbO}_{6}$ is due to the high stability of the NTO-type structure at high temperatures. The effect of this secondary phase on the magnetic behaviour of $\mathrm{DPv} \_\mathrm{Mn}_{2} \mathrm{ScSbO}_{6}$ is discussed below.

Fig. 5b depicts the nuclear (and magnetic) structure of $\mathrm{DPv}_{-} \mathrm{Mn}_{2} \mathrm{ScSbO}_{6} ; \mathrm{Sb}$ and $\mathrm{Sc}$ are six-fold coordinated and ordered in a rock-salt configuration. Mn cations occupy the highly distorted cuboctahedral voids. The tilt angle, calculated as $\Phi=\left(180^{\circ}-\theta\right) / 2$ from $<\mathrm{B}-\mathrm{O}-\mathrm{B}^{\prime}>$ bond angles (see Table 2$)$ is $\Phi=21.0$ (3) $)^{\circ}$ This value is indicative of the high degree of distortion attained in this polymorph. The polyhedral distortions $(\Delta$ in Table 2$)$ are also abnormally large $\left(\Delta\left[\mathrm{ScO}_{6}\right]=2.9 \times 10^{-4}\right.$ and $\left.\Delta\left[\mathrm{SbO}_{6}\right]=1.6 \times 10^{-5}\right)$, close to those observed in other perovskites containing small-sized cations as $\mathrm{Mn}^{2+}$ and $\mathrm{Sc}^{3+}$ at the A site, as $\mathrm{MnVO}_{3}$ or $\mathrm{ScVO}_{3}$ with $\Phi=18.02^{\circ}$ and $26^{\circ}$ and $\Delta=$ $4.4 \times 10^{-4}$ and $4.0 \times 10^{-4}$ respectively. ${ }^{5,26}$ The calculated BVS values are close to the expected values for the nominal composition $\mathrm{Mn}_{2}^{2+}{ }_{2} \mathrm{Sc}^{3+} \mathrm{Sb}^{5+} \mathrm{O}_{6}$.

The microstructure of this double perovskite is clearly observed in the HRTEM image recorded along the [010] zone axis (see SF2c $\dagger$ ). The image and its corresponding FFT indicate the absence of extended defects and a good agreement with the calculated image is found. EELS experiments performed on the $\mathrm{Mn}$ L-edge in several crystals of $\mathrm{DPv}_{-} \mathrm{Mn}_{2} \mathrm{ScSbO}_{6}$ revealed an average oxidation state of +2 for $\mathrm{Mn}$, in correspondence with BVS calculations.

Magnetic behaviour and magnetic structure. The ZFC and FC (black and red circles, respectively) molar magnetic susceptibilities for the $\mathrm{DPv} \_\mathrm{Mn}_{2} \mathrm{ScSbO}_{6}$ polymorph and the reciprocal susceptibility (open circles) as a function of temperature under an applied field of $3 \mathrm{kOe}$ are depicted in Fig. 5c. It follows the Curie-Weiss behaviour with an additional $0.0579 \mathrm{emu}^{-1} \mathrm{~mol}$ Oe temperature independent paramagnetic (TIP) contribution

Table 2 Crystallographic parameters, selected interatomic distances (Å) and angles $\left(^{\circ}\right)$ for $\mathrm{DPv} \_\mathrm{Mn}_{2} \mathrm{ScSbO}{ }_{6}$ from the room temperature NPD Rietveld refinement in the $P 2_{1} / n$ space group ${ }^{a}$

\begin{tabular}{|c|c|c|c|c|c|}
\hline $\mathrm{Sc} / \mathrm{Mn}$ & $2 d$ & $\frac{1}{2}$ & 0 & 0 & $0.909(4) / 0.091(4)$ \\
\hline $\mathrm{Sb}$ & $2 \mathrm{c}$ & ${ }_{0}^{2}$ & $\frac{1}{2}$ & 0 & 1 \\
\hline $\mathrm{O} 1$ & $4 e$ & $0.313(2)$ & $0.321(2)$ & $0.940(1)$ & 1 \\
\hline $\mathrm{O} 2$ & $4 \mathrm{e}$ & $0.327(2)$ & $0.307(2)$ & $0.570(1)$ & 1 \\
\hline$\left(\mathrm{B}-\mathrm{O}_{2}\right) \times 2$ & $2.10(1)$ & $\left(\mathrm{Sb}-\mathrm{O}_{2}\right) \times 2$ & $1.99(1)$ & $\Delta\left[\mathrm{BO}_{6}\right]$ & $2.9 \times 10^{-4}$ \\
\hline$\left(\mathrm{B}-\mathrm{O}_{3}\right) \times 2$ & $2.15(1)$ & $\left(\mathrm{Sb}-\mathrm{O}_{3}\right) \times 2$ & $2.00(1)$ & $\Delta\left[\mathrm{SbO}_{6}\right]$ & $1.6 \times 10^{-5}$ \\
\hline$<\mathrm{A}-\mathrm{O} 1-\mathrm{A}>$ & $113(1)$ & $<\mathrm{A}-\mathrm{O} 2-\mathrm{A}>$ & $104(1)$ & $<\mathrm{A}-\mathrm{O} 3-\mathrm{A}>$ & $123(1)$ \\
\hline$<\mathrm{B}-\mathrm{O} 1-\mathrm{B}^{\prime}>$ & $140.9(3)$ & $<\mathrm{B}-\mathrm{O} 2-\mathrm{B}^{\prime}>$ & $137.1(3)$ & $<\mathrm{B}-\mathrm{O} 3-\mathrm{B}^{\prime}>$ & $136.8(3)$ \\
\hline $\mathrm{BVS}(\mathrm{Mn})^{b}$ & 1.7 & BVS (Sc) & 2.9 & BVS (Sb) & 5.3 \\
\hline
\end{tabular}

${ }^{a}$ Fitting residuals: $R_{\mathrm{p}}=2.00 \%, R_{\mathrm{wp}}=2.51 \%, R_{\mathrm{B}}=6.04 \%$ and $R_{\mathrm{F}}=4.36 \% .{ }^{b} V_{i}=\sum_{j} S_{i j} i=\exp \left(r_{0}-r_{i j} / 0.37\right)$. Values calculated using $r_{i j}=1.79 \AA$ for $\mathrm{Mn}^{2+}, 1.849 \AA$ for $\mathrm{Sc}^{3+}$ and $1.942 \AA$ for $\mathrm{Sb}^{5+}$. Polyhedral distortions calculated from $\Delta=1 / n \times \sum\left[\left(d_{i}-d_{\mathrm{av}}\right) / d_{\mathrm{av}}\right]^{2}$. 
above $100 \mathrm{~K}$. The Weiss temperature is $\theta_{\mathrm{DPv}}=-94 \mathrm{~K}$ and the effective paramagnetic moment $\mu_{\mathrm{DPv}}=5.5 \mu_{\mathrm{B}}$ per $\mathrm{Mn}^{2+}$ is slightly lower than the expected value for $S=5 / 2 \mathrm{Mn}^{2+}$ $\left(5.92 \mu_{\mathrm{B}}\right)$. The magnetic susceptibility increases abruptly at $\sim 42 \mathrm{~K}$ due to the presence of the NTO polymorph as a secondary phase. This obscures the antiferromagnetic transition at 22.3 K, determined below for this polymorph from NPD. The absence of magnetic cations neither in B nor in $\mathrm{B}^{\prime}$ - sites yields the only possible magnetic interactions in this compound to be those operating between the nearest neighboring $\mathrm{Mn}^{2+}$ cations. The long $\mathrm{Mn}-\mathrm{Mn}$ distance and the high coordination of the A - site, favors the development of cooperative superexchange interactions through oxygen anions. The nature and strength of these interactions is defined by the $\mathrm{Mn}-\mathrm{O}-\mathrm{Mn}$ angles, which take the values of $104(1)^{\circ}, 113(1)^{\circ}$ and $123(1)^{\circ}$. According to the Goodenough-Kanamori rules ${ }^{27}$ these angles rule the antiferromagnetic interactions among $\mathrm{d}^{5}$ cations, as observed in the magnetization curves (see SF3† bottom). From the Rietveld refinement, it has been found that 9.1\% of A-sites are occupied by diamagnetic $\mathrm{Sc}^{3+}$ cations, resulting in a magnetic dilution effect on the overall $\mathrm{Mn}^{2+}$ sublattice.

The magnetic structure of $\mathrm{DPv} \_\mathrm{Mn}_{2} \mathrm{ScSbO}_{6}$ was determined by NPD using data from the D1B instrument. Additional magnetic neutron diffraction peaks are observed below $22 \mathrm{~K}$.

Fig. 5a (bottom) shows the fit to the $3 \mathrm{~K}$ data. The magnetic reflections can be indexed with the $k=\left[\begin{array}{lll}0 & 0 & 0\end{array}\right]$ propagation vector in the $P 2_{1} / n$ space group. Irreducible representations and their basis vectors were obtained by using the program BASIREPS. Fig. 5b shows the resulting magnetic structure. It can be described as an antiparallel orientation of $\mathrm{Mn}^{2+}$ spins located in the ac-plane, with a total moment of 4.54 (1) $\mu_{\mathrm{B}}$ at $3 \mathrm{~K}$. The deviation of the spins with respect to the $c$ axis has been calculated to be $\gamma=20.34(1)^{\circ}$, resulting in 1.58 (1) $\mu_{\mathrm{B}}$ and $4.26(1) \mu_{\mathrm{B}}$ components along the $a$ - and $c$-axes respectively. The inset in Fig. 5a shows the thermal evolution of the ordered magnetic moment, fitted to the critical law. The fitting results in $T_{\mathrm{N}}=22.3 \mathrm{~K}$ and $\beta=0.37$, where $\beta$ agrees again with 3D Heisenberg antiferromagnetic behaviour.

\section{Conclusions}

Our results demonstrate that two new phases of $\mathrm{Mn}_{2} \mathrm{ScSbO}_{6}$ are accessible by high pressure-high temperature synthesis and their structural and microstructural details and magnetic properties have been investigated.

Although cations are completely randomly located within the room pressure polymorph, frustrating any long range magnetic order, the synthesis of this oxide under high pressure conditions induces different cationic arrangements leading to interesting properties. The NTO-type moderate pressure modification crystallizes in the rhombohedral $R 3$ polar space group, which allows a predicted room temperature polarization of $28.3 \mu \mathrm{C} \mathrm{cm}^{-2}$. It has ferrimagnetic order below $42 \mathrm{~K}$, with the spins lying in the $a b$ plane. A ferromagnetic component of $0.6 \mu_{\mathrm{B}}$ has been determined to arise from unusual site-selective
$\mathrm{Mn} / \mathrm{Sc}$ disorder. NTO_ $\mathrm{Mn}_{2} \mathrm{ScSbO}_{6}$ is thus a potential multiferroic material. The high pressure phase has a double perovskite structure crystallizing in the $P 2_{1} / n$ space group, which exhibits an antiferromagnetic order below $22.3 \mathrm{~K}$. Its magnetic structure has antiparallel $\mathrm{Mn}^{2+}$ spins in the $a c$ plane.

\section{Acknowledgements}

The authors thank MINECO and Comunidad de Madrid for foundation through projects MAT2013-44964 and S-2013/ MIT-12753, respectively. EPSRC and the Royal Society are also acknowledged for support, and Dr Gallardo-Amores and Dr Romero-de Paz for HPHT synthesis and magnetic measurements.

\section{Notes and references}

1 V. Srivastava, M. Rajagopalan and S. P. Sanyal, Eur. Phys. J. B, 2008, 61, 131.

2 R. J. Harrison, U. Becker and S. A. T. Redfern, Am. Mineral., 2000, 85, 1694.

3 K. Leinenweber, Phys. Chem. Minerals, 1991, 18, 244.

4 L. C. Ming, Y. H. Kim, T. Uchida, Y. Wang and M. Rivers, Am. Mineral., 2006, 91, 120.

5 M. Markkula, A. M. Arevalo-Lopez, A. Kusmartseva, J. A. Rodgers, C. Ritter, H. Wu and J. P. Attfield, Phys. Rev. B: Condens. Matter, 2011, 84, 094450.

6 A. M. Arévalo-López and J. P. Attfield, Phys. Rev. B: Condens. Matter, 2013, 88, 104416.

7 S. Vasala and M. Karppinen, Prog. Solid State Chem., 2015, 43, 1.

8 R. H. Mitchell, Perovskites: modern and ancient, Almaz Press Inc., 2002.

9 M. R. Li, D. Walker, M. Retuerto, T. Sarkar, J. Hadermann, P. W. Stephens, M. Croft, A. Ignatov, C. P. Grams, J. Hemberger, I. Nowik, P. S. Halasyamani, T. T. Tran, S. Mukherjee, T. S. Dasgupta and M. Greenblatt, Angew. Chem., Int. Ed., 2013, 52, 8406.

10 M. R. Li, M. Retuerto, D. Walker, T. Sarkar, P. W. Stephens, S. Mukherjee, T. S. Dasgupta, J. P. Hodges, M. Croft, C. P. Grams, J. Hemberger, J. Sánchez-Benítez, A. Huq, F. O. Saouma, J. I. Jang and M. Greenblatt, Angew. Chem., Int. Ed., 2014, 53, 10774.

11 M. R. Li, M. Croft, P. W. Stephens, M. Ye, D. Vanderbilt, M. Retuerto, Z. Deng, C. P. Grams, J. Hemberger, J. Hadermann, W. M. Li, C. Q. Jin, F. O. Saouma, J. I. Jang, H. Akamatsu, V. Gopalan, D. Walker and M. Greenblatt, Adv. Mater., 2015, 27, 2177.

12 A. J. Dos santos-García, C. Ritter, E. Solana-Madruga and R. Sáez-Puche, J. Phys.: Condens. Matter., 2013, 25, 206004.

13 A. J. Dos santos-García, E. Solana-Madruga, C. Ritter, D. Ávila-Brande, O. Fabelo and R. Sáez-Puche, Dalton Trans., 2015, 44, 10665. 
14 S. Ivanov, P. Nordblad, R. Mathieu, R. Tellgren, E. Politova and G. André, Eur. J. Inorg. Chem., 2011, 4691.

15 L. I. Kosse, E. D. Politova and V. V. Chechkin, Izv. AN SSSR, Neorg. Mater., 1982, 18, 1879.

16 L. I. Kosse, E. D. Politova and Yu. N. Venevtsev, Zh. Neorg. Khim., 1983, 28, 1689.

17 Mac Tempas X. Version 2.3.7.A program for simulating HRTEM images and diffraction patterns.

18 H. K. Schmid and W. Mader, Micron, 2006, 37, 426.

19 J. Rodriguez-Carvajal, Physica B, 1993, 192, 55.

20 J. Rodriguez-Carvajal, BASIREPS: a program for calculating irreducible representations of space groups and basis functions for axial and polar vector properties. Part of the FullProf Suite of programs, http://www.ill.eu/sites/fullprof/.
21 J. P. Morniroli and J. W. Steeds, Ultramicroscopy, 1992, 45, 219.

22 S. A. Ivanov, R. Mathieu, P. Nordblad, R. Tellgren, C. Ritter, E. Politova, G. Kaleva, A. Mosunov, S. Stefanovich and M. Weil, Chem. Mater., 2013, 25, 935.

23 N. A. Spaldin, J. Solid State Chem., 2012, 195, 2.

24 P. D. Battle, M. Avdeev and J. Hadermann, J. Solid State Chem., 2014, 220, 163.

25 G. V. Bazuev, B. G. Golovkin, N. V. Lukin, N. I. Kadyrova and Yu. G. Zainulin, J. Solid State Chem., 1996, 124, 333.

26 E. Castillo-Martínez, M. Bieringer, S. P. Shafi, L. M. D. Cranswick and M. A. Alario-Franco, J. Am. Chem. Soc., 2011, 133, 8552.

27 J. B. Goodenough, Magnetism and the chemical bond. Interscience monographs on chemistry, 1963. 